\title{
Insertion Device Vacuum Chamber for the ELETTRA Storage Ring
}

\author{
J. MIERTUSOVA, N. PANGOS, Sincrotrone Trieste, Padriciano 99, 34012 Trieste Italy
}

\section{Abstract}

The first four insertion device (ID) vacuum chambers (3 for the undulators, 1 for the wiggler) have been installed in the ELETTRA synchrotron radiation facility. Each chamber is 4.8 $\mathrm{m}$ in length and has a rectangular cross section with a cylindrical antechamber. The whole system is pumped by four $120 \mathrm{l} / \mathrm{s}$ sputter-ion pumps of nominal pumping speed. The insertion device chambers are fabricated from stainless steel ESR AISI $316 \mathrm{LN}$. This material was chosen for its very low relative magnetic permeability $(\sim 1.003)$, its high yield stress and the well known procedures for welding and cleaning. Vacuum and mass spectroscopy experience during the first year of commissioning is presented.

\section{INTRODUCTION}

Elettra synchrotron radiation facility has been built expecially for the use of high brilliance radiation from insertion devices ID. The storage ring of $260 \mathrm{~m}$ in circumference is equipped by 12 straight sections, one is for beam injection, 11 are for IDs beam lines.

An austenitic stainless steel ESR AISI 316 LN has been chosen as the material of the vacuum chamber, for its low magnetic permeability, high yield stress and well known welding procedure. To minimise synchrotron radiation induced desorption as well as thermal outgassing. All vacuum components were carefully cleaned and finished.

In this paper we present a series of measurements of the outgassing rate, vacuum performances during operation (gap closure at different beam currents and energies) and results of the mass spectroscopy scanning. Special attention was paid to manufacture the ID vacuum chamber, all steps of production were carefully checked by the Quality control group of Sincrotrone Trieste.

\section{VACUUM CHAMBER DESIGN AND MANUFACTURE}

Firstly we planed to use the NEG St 707 strips to obtain and to maintain UHV conditions in the ID vacuum chamber. However, the NEG activation at $450{ }^{\circ} \mathrm{C}$ performed serious problems due to the thermal expansion of the NEG strips. In fact, some short circuits were formed between the NEG and the chamber walls. The theoretical simulation of the partial pressures of methane and noble gases (especially Argon) have also shown a significant pressure increase, because of a negligible pumping speed of the NEG for these gases.

The ID vacuum chamber was designed in our laboratory in collaboration with the technical office. It has a rectangular cross-section with a cylindrical ante-chamber, as it is shown in fig. 1 .

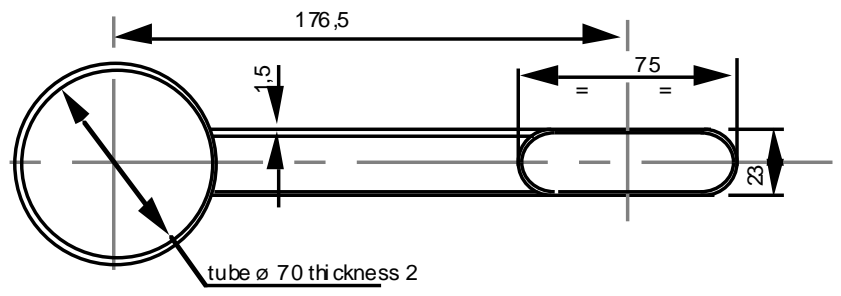

fig. 1 - Cross-section of the ID vacuum chamber

The whole system is pumped by four 120 1/s SIPs, two pumps are installed on both ends of the chamber, the other two are proportionally placed in between. The expected pressure profile which should be obtained after about $50 \mathrm{Ahs}$ of conditioning is presented in fig. 2, the minimum values in this graph correspond to the SIPs position [2].

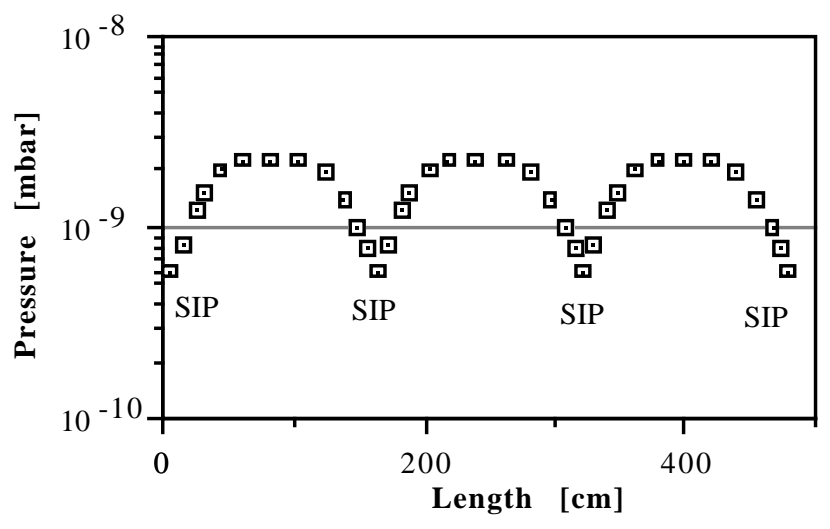

fig. 2 - Theoretical pressure profile in the ID chamber

Special care was gives to fabricate and clean all stainless steel components. The fabrication procedure was following the norm ASME II, the welding procedure was performed according to the norm ASME IX and radiographic control was made following the norm ASME V. All components were assembled and welded in a clean room. More details can be found in ref. 1.

Internal surfaces and welds exposed to the vacuum must be free of micro inclusions and cracks. Surface roughness must be less than $1 \mu \mathrm{m}$ and free of oxides and impurities. The ID vacuum chamber (without the end flanges) was carefully cleaned and finished to obtain the required vacuum level $\left(<1 \times 10^{-10}\right.$ mbar without the beam). 
A complete surface treatment included the following phases: organic solvent degreasing, ultrasonic washing, two phases rinsing (in normal and in demineralized water), drying. The cleaned ID vacuum chamber was than pre-baked and degassed in our high vacuum oven. Pre baking was performed at $350{ }^{\circ} \mathrm{C}$ for 24 hours, and at the end the pressure in the oven decreased down to $1 \times 10^{-7}$ mbar range.

Before twelding the flanges, the chamber was treated at temperature of $450{ }^{\circ} \mathrm{C}$ for 2 hours in an innert gases atmosphere to stretch all welds of the antechamber.

\section{OUTGASSING RATE AND MASS SPECTRA}

The specific outgassing rate of the ID vacuum chamber was measured and verified at different well defined vacuum conditions. To evaluate the specific outgassing rate $q_{D}$ the expression

$$
\mathrm{q}_{\mathrm{D}}=\left(\mathrm{P}_{1}-\mathrm{P}_{2}\right) 2 \mathrm{C} / \mathrm{B} \mathrm{L}
$$

where $\mathrm{P}_{1}$ and $\mathrm{P}_{2}$ are pressures at both ends of the chamber, $\mathrm{B}$ and $\mathrm{L}$ are the perimeter and length of the chamber, respectively, $\mathrm{C}$ is the conductance in $1 / \mathrm{s}$.

After delivery, the chamber was equipped with the two central SIPs, on the one side the turbo-pump Seiko was installed, and the opposite end was connected with the forevacuum pump. After 6 hours of pumping the equilibrium pressure of $2.6 \times 10^{-6}$ mbar and $1.2 \times 10^{-6}$ mbar was reached at the ends of the chamber. In the mass spectrum scanned at this condition, the mass peaks of hydrocarbons and water were enormous. The corresponding specific outgassing rate was $6 \times 10^{-10} \mathrm{mbar} .1 / \mathrm{s} . \mathrm{cm}^{2}$.

Then the ID vacuum chamber was baked-out at $230{ }^{\circ} \mathrm{C}$ for 36 hours. At the maximum temperature the pressure exceeded the $10^{-5}$ mbar leveld.

In fig. 3 the mass spectrum scanned at the temperature of $175^{\circ} \mathrm{C}$ is shown.

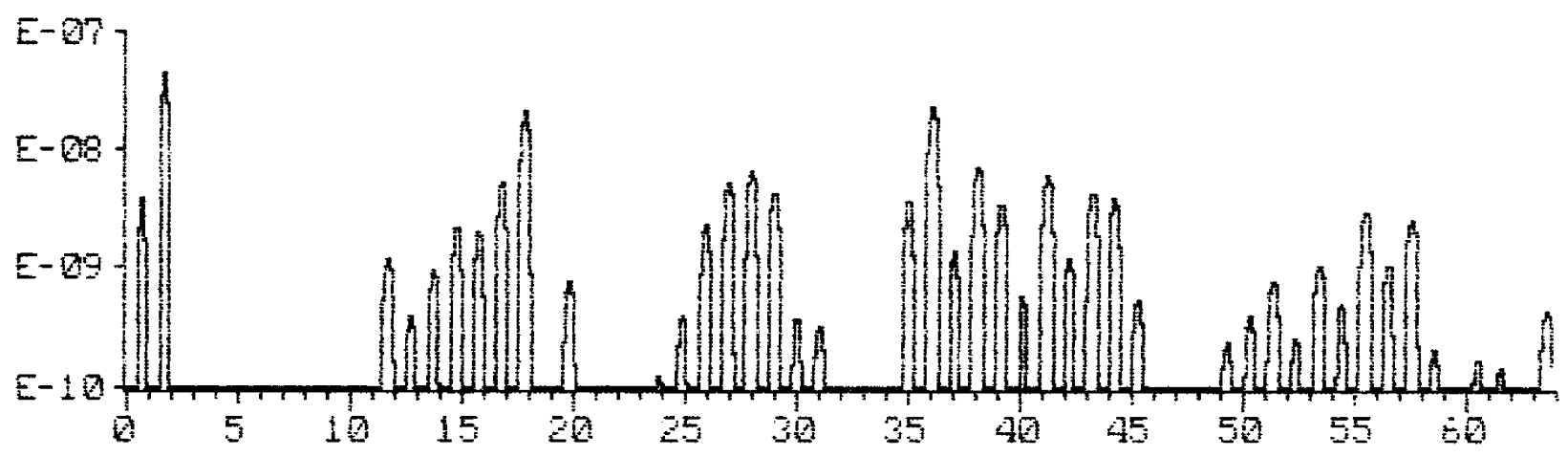

fig. 3 - Mass spectrum scanned at $175^{\circ} \mathrm{C}$

In spite of carefully performed cleaning the hydrocarbons and water peaks were still present. Only after prolonging the bake-out and the subsequent cooling, these unwanted peaks disappeared and the ultimate pressure of $1.2 \times 10^{-9}$ mbar was achieved. The corresponding outgassing rate was then $2 \times 10^{-12}$ mbar. $1 / \mathrm{s} . \mathrm{cm}^{2}$. After the baking procedure all the dimensions of the chamber were precisely measured, the chamber was perfectly alligned with the acceptable tolerance of $0.2 \mathrm{~mm}$.

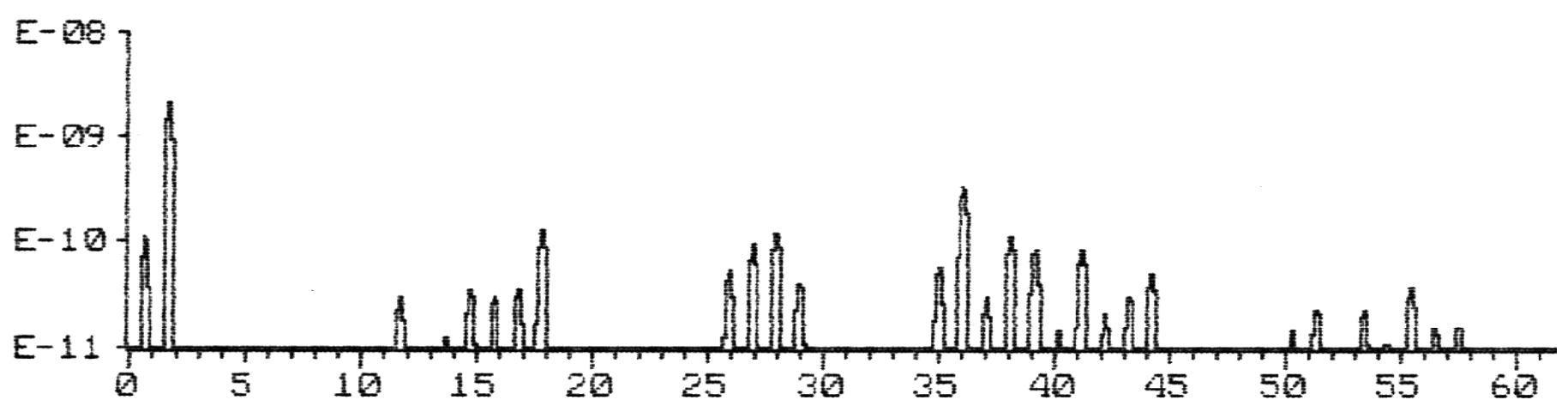

fig. 4 - Mass spectrum scanned at room temperature

The ID vacuum chamber heated and degassed in this manner, was than saturated by dry nitrogen, closed with the flanges and transported into the tunnel.
The installation of the chamber in the storage ring was done under a continuous flow of dry nitrogen, and a bake-out in situ was performed. 
During the in situ bake-out the maximum temperature of $180{ }^{\circ} \mathrm{C}$ was kept for 36 hours. When the chamber was cold and through the ID valves connected with the rest of the ring, the equilibrium pressure in the $10^{-10}$ mbar range was maintained. The specific outgassing rate in $10^{-13} \mathrm{mbar} .1 / \mathrm{s} . \mathrm{cm}$ range was achieved and a very clean vacuum was obtained see fig. 5

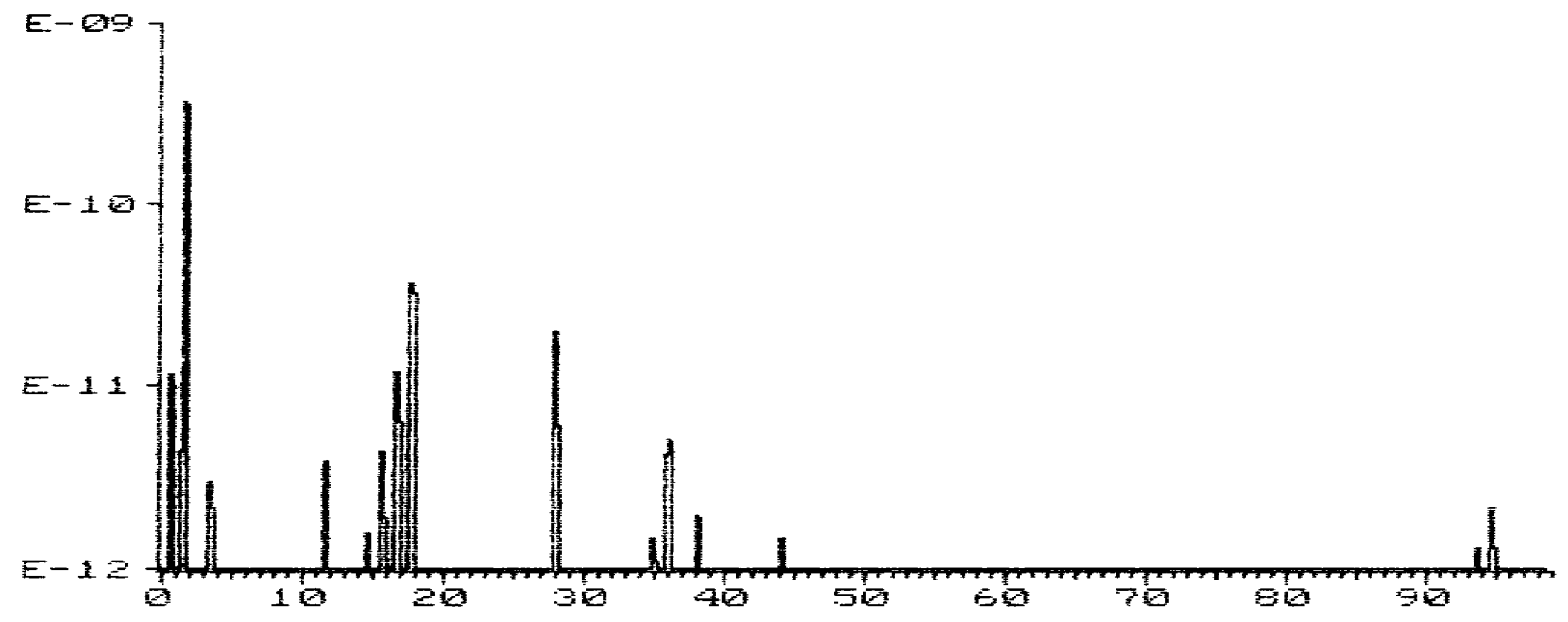

fig. 5 - Mass spectrum at the equilibrium pressure of 3.6e-10 mbar in the ring

The ID chamber conditioning has been done in two steps: at the beginning the gap of the ID magnets was opened to the maximum. The pressure in the chamber with increasing beam current firstly exceeded more than one decade the pressure in the rest of the ring. After about 30 Ahs of conditioning the pressure profile became uniform. The second step of conditioning started by closing the gap of the ID magnets. Especially in case of the wiggler the photodesorption was very pronounced and it caused lifetime drop as shown in fig. 6.

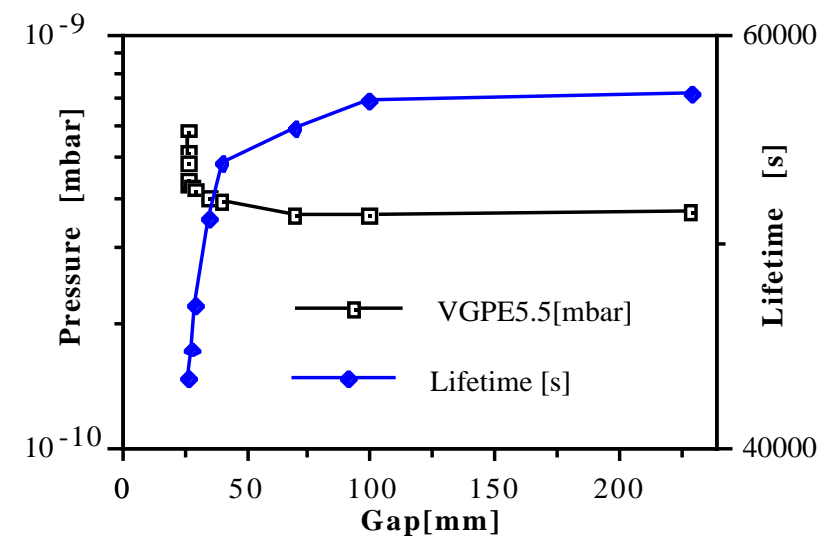

fig. 6 - Pressure and lifetime dependence in the wiggler during the gap closure

This effect was be observed only for a short time and disappeared after 3-5 of the gap closures (also at higher currents and energies). The pressure in the ID chamber then became lower than in the rest of the ring.

\section{CONCLUSIONS}

For the vacuum performance of our ID vacuum chamber, can be concluded:

1. it is very important to perform careful manufacturing and cleaning of the ID vacuum chamber, following the technical norms and standards,

2. the ultimate pressure in the $10^{-10}$ mbar range can be achieved after an in situ bake-out, the desorption yield of $1.5 \times 10^{-6} \mathrm{~mol} /$ photon can be obtained after approximately 30 Ahs of conditioning at $2 \mathrm{GeV}$,

3. no special difficulties are expected from the vacuum point of view with the small gap ID vacuum chamber - $15 \mathrm{~mm}$ vertically - which will be installed in the near future.

\section{REFERENCES}

[1] N. Pangos: Prescrizione di fornitura per la fabricazione della camera da vuoto per ID di ELETTRA, Technical Note N. S044, Sincrotrone Trieste S.P.A., 1993

[2] J. Miertusova: Theoretical Simulation of the Pressure Profile in the ID Vacuum Chamber, Technical Note, Sincrotrone Trieste, S. P. A., 1993 